\title{
When trying to recall our past, all roads lead to Rome: More evidence for the multi-process retrieval theory of autobiographical memory
}

\author{
John H. Mace ${ }^{1}$ - Bobbie Jo A. Staley ${ }^{1} \cdot$ McKenzie K. Sopoci $^{1}$ \\ Accepted: 15 September 2020 / Published online: 25 September 2020 \\ (C) The Psychonomic Society, Inc. 2020
}

\begin{abstract}
In this study, we tested elements of the multi-process retrieval account of autobiographical memory, retrieval multiplicity, and retrieval selectivity. The retrieval multiplicity states that multiple different retrieval strategies are used to recall autobiographical memories, while the retrieval selectivity states that such retrieval strategies will vary in accord with the cuing environment. We tested these hypotheses across two experiments with the retrieve-aloud procedure. In the retrieve-aloud procedure, participants were required to verbalize their thoughts while attempting to retrieve personal memories in response to phrase cues (e.g., listening to music). Experiments 1 and 2 found support for the retrieval multiplicity by showing that participants used a variety of different retrieval processes (eight different processes in total), while Experiment 1 found support for the retrieval selectivity by showing that retrieval strategies varied across different cue types. The implications of the findings are discussed with respect to autobiographical memory, as well as semantic memory.
\end{abstract}

Keywords Autobiographical memory retrieval $\cdot$ Retrieval strategies $\cdot$ Multi-process retrieval theory $\cdot$ Direct and generative retrieval $\cdot$ Voluntary autobiographical memories $\cdot$ Autobiographical memory

\section{Introduction}

Attempts to understand how we intentionally recall the past have a fairly long history in autobiographical memory research. Norman and Bobrow (1979) argued that retrieval from autobiographical memory was a cyclical process, where one cycles through stages of cue elaboration, memory search, and evaluation until the sought-after memory is found (see also Williams \& Hollan, 1981). In more recent history, Conway and colleagues (e.g., Conway, 2005; Conway \& PleydellPearce, 2000) proposed that two distinctly different types of retrieval processes are at work during intentional recall: direct retrieval - where cues appear to map directly onto to matching episodic memories without effort, and generative retrieval where effortful searches proceed through the hierarchical layers of autobiographical memory (i.e., from lifetime periods to general events to ultimately episodic memories) (see Haque \& Conway, 2001; Haque, Juliana, Khan, \& Hasking, 2014;

John H. Mace

jhmace@eiu.edu

1 Psychology Department, Eastern Illinois University, Charleston, IL 61920, USA
Williams, Chan, Crane, \& Barnhofer, 2006). Uzer, Lee, and Brown (2012) present an alternative two-process account, where direct retrieval is viewed as the dominant form, and generative retrieval is viewed as processes where cues are generated in the service of retrieving memories. Mace, Clevinger, Delaney, Mendez, and Simpson (2017) proposed that intentional recall in autobiographical memory involves multiple retrieval processes, direct and hierarchical retrieval (as described by Conway and colleagues for generative retrieval), as well as a temporal recall strategy (where ones uses generic temporal cues, such as the days of the week), and a repeating strategy (where one continuously repeats the cue until a memory comes to mind).

Thus, researchers have viewed autobiographical memory retrieval as a single process (e.g., Norman \& Bobrow, 1979), a dual process (e.g., Haque \& Conway, 2001, and Uzer et al., 2012, although these views differ greatly from one another), or a multi-process (Mace et al., 2017). In the current study, we extend the multi-process retrieval theory. Before we outline the details of the current study, we first review the multiprocess retrieval theory in greater detail, as well as the supporting evidence and general findings in Mace et al. (2017), and we compare this view to other current views on autobiographical memory retrieval. 
The multi-process retrieval theory states that intentional autobiographical memory recall involves a number of different recall processes, and that individuals will routinely use or select among a number of different retrieval strategies a process that may be driven in part by the circumstances of retrieval (e.g., the characteristics of a cue) and their meta-awareness of the processes of remembering (for support of these principles, see Barzykowski, Niedzwienska, \& Mazzoni, 2019; Barzykowski \& Staugaard, 2018; Uzer, 2016; Uzer \& Brown, 2017). At a minimum, the processes involve direct retrieval, hierarchical retrieval, temporal retrieval, and repeating retrieval. With the exception of direct retrieval, all of these retrieval processes are mental strategies that individuals can select from or switch back and forth from (a process we call mixed strategies). Direct retrieval is generally not considered a strategy because it occurs quickly (typically in 3-5 s, e.g., Barzykowski \& Staugaard, 2016; Haque \& Conway, 2001; Mace et al., 2017; Uzer, 2016; Uzer et al., 2012), and it appears to occur without any overt mental strategy (e.g., cue elaboration), much like in the case of everyday involuntary autobiographical remembering (e.g., Ball \& Little, 2006; Berntsen, 1996, 1998; Mace, 2005; Schlagman \& Kvavilashvili, 2008; see reviews in Conway, 2005). ${ }^{1}$ In contrast, strategic processes involve relatively more effort, and each has a different identifiable mental process. For example, it would be obvious to an individual (or a third party if retrieval occurs out loud) that one is using the hierarchal, temporal, or repeating strategy by examining the nature of one's mental contents (i.e., by witnessing that one is running through lifetime periods, using general temporal cues, or simply repeating a cue), while in contrast direct retrieval is inferred by the apparent lack of a mental strategy. Given their temporal foundations (either personal or general), both hierarchal and temporal retrieval are processes that are sensibly related to the recall of autobiographical memory (e.g., Haque \& Conway, 2001; Thompson, Skowronski, \& Betz, 1993). Repeating, while having no obvious relationship to autobiographical memories, is a process that may be commonly used when one is trying to recall general semantic (e.g., what is the capitol of Somalia) or personal semantic (e.g., what was the name of my fifth grade teacher) information. Direct retrieval appears to underlie many forms of memory (e.g., autobiographical, semantic, and many forms of implicit memory).

Thus, the multi-process retrieval theory argues that autobiographical memory retrieval is highly versatile, as there are

\footnotetext{
${ }^{1}$ The characterization of direct retrieval as non-strategic recall may best fit involuntary autobiographical remembering. Direct retrieval in voluntary remembering should at least involve the processing of retrieval cues as items potentially related to one's past (i.e., retrieval-mode processing; Tulving, 1983) (for more on direct retrieval in voluntary and involuntary recall, see Barzykowski \& Staugaard, 2016; Barzykowski et al., 2019; Harris \& Berntsen, 2019; Uzer et al., 2012). Thus, direct retrieval may be best characterized as a retrieval process with minimal effort and strategy.
}

numerous ways in which one can retrieve (or construct) autobiographical memories. This notion makes sense when one considers the rich and complex nature of autobiographical memories. Indeed, retrieval versatility may be a basic property of declarative memory (e.g., see Minakova \& Falikman, 2011, where multiple retrieval strategies were used when participants were searching for semantic memories). In contrast, retrieval in non-declarative (or implicit) memory appears to be less versatile, as these forms of memory appear to be governed by direct retrieval alone (see Roediger \& McDermott, 1993; Schacter, 1992).

Mace et al. (2017) found support for the multi-process retrieval theory with the retrieve-aloud procedure (Uzer et al., 2012). In this approach, participants were given retrieval cues (phrases, such as listening to music) with the instructions to recall specific autobiographical memories (i.e., episodic memories) matching the cues, following standard autobiographical memory-testing practices. However, departing from standard practices, participants were further instructed to retrieve memories out loud, verbalizing all of the contents of their thoughts, beginning with the onset of the retrieval cue and continuing until a memory came to mind and was verbalized. The retrieval portions of these verbal protocols were then categorized according to retrieval type (e.g., direct, hierarchical, etc.). Analyses of the retrieval types in the first experiment of that study showed participants had used both direct and hierarchical retrieval, as in previous reports (e.g., Haque \& Conway, 2001). Most crucially, participants were also found to use the repeating- and temporal-retrieval strategies, as well as mixed strategies, thereby supporting the multi-process retrieval theory. With respect to frequency, direct retrieval had the highest prevalence (57\%, consistent with Uzer, 2016, Uzer et al., 2012, and Barzykowski \& Staugaard, 2016), mixed strategies the lowest (1\%), while hierarchical, repeating, and temporal strategies occurred with equal frequency (ranging from 12\% to $15 \%$ ). In a second experiment, participants were divided into groups where they were instructed to use the hierarchical, repeating, or temporal strategies to recall autobiographical memories. The results of this experiment showed that repeating was the fastest strategy to produce memories (some $5 \mathrm{~s}$ vs. 8-9 s for temporal and hierarchical), while temporal was the strategy most likely to fail in memory production (11\% failures vs. 5\% in hierarchical and repeating). The results of this experiment demonstrated that each of the different strategies have distinctly different characteristics and outcomes, as repeating differed from the hierarchical and temporal strategies in terms of retrieval speed, and hierarchical and temporal differed from one another in terms of retrieval success. Such data help confirm the proposition that the strategies are truly involved in retrieval, and that they distinctly differ from one another.

In addition to the evidence presented in Mace et al. (2017), there are a number of other studies that are in some way 
supportive of a multi-process retrieval account. For example, studies have shown that individuals use a number of retrieval strategies when they are searching for semantic memories (as noted above, Minakova \& Falikman, 2011), or retrieving words on explicit and implicit memory tasks (e.g., Mace 2003; Richardson-Klavehn, Gardiner, \& Java, 1994). In the autobiographical memory literature, Burt, Kemp, and Conway (2001) found that participants used multiple strategies when they were attempting to retrieve the dates of events. More directly, data from studies supporting the dual-process account (e.g., Uzer et al., 2012) are also in line with the multi-process view, as this approach views generative retrieval as involving different cuegeneration strategies (discussed below).

How does the multi-process view differ from other contemporary accounts of autobiographical memory retrieval? In Conway's view (e.g., Conway, 2005; Haque \& Conway, 2001), autobiographical memory recall involves either direct retrieval or generative retrieval (i.e., hierarchical retrieval), where the latter is viewed as the dominant form. In Uzer et al.'s (2012) dual-process account, retrieval from autobiographical memory is viewed as either involving direct retrieval or generative retrieval, where the former is seen as dominant. In addition, unlike Conway's view on generative retrieval, the dual-process account views generative retrieval as involving different cue-generation retrieval strategies, rather than solely involving hierarchical retrieval. Thus, the multiprocess view is similar to the dual-process account, except that the former focuses more on explicating the different retrieval strategies, and the dual-process view also speaks to the nature of the representational form of autobiographical memories (i.e., stable and retrieved versus transitory and constructed; for more on these views, see Conway, 2005; Uzer et al., 2012; and also Harris, O'Connor, \& Sutton, 2015, who combine both views). The multi-process view is neutral on the constructed versus retrieved debate (e.g., Haque \& Conway, 2001; Harris et al., 2015; Uzer et al., 2012), as either view can be fit to a multi-strategy approach. Relatedly, while the dualprocess theory views strategies as involving cue generation, the multi-process theory views strategies as involving either cue generation or memory-content generation (e.g., particularly in the case of hierarchical retrieval; for similar views, see also Harris et al., 2015; Harris \& Berntsen, 2019).

Concerning the mechanisms of memory production, the multi-process account views each retrieval strategy as a distinct mental process that is capable of generating autobiographical memories. For example, in hierarchical retrieval one generates lifetime period knowledge and general-event memories, which in turn may be elaborated into episodic memories (Conway, 2005). Similarly, in temporal retrieval one uses generic temporal cues (e.g., days of the week or seasons of the year) in order to activate episodic memories, and this process may at times overlap with hierarchical retrieval if one generates general autobiographical memories, though this process as a whole appears to be less effective than hierarchical retrieval (Mace et al., 2017). Perhaps spreading activation underlies both of these processes, which may explain why temporal retrieval is less successful, as generic temporal cues may be imperfectly associated with episodic memories. Direct retrieval appears to be a very different mental process, as memories appear to be generated simply by focusing one's attention on a retrieval cue. We believe that the repeating strategy is an extension of direct retrieval, as repeating appears to be a strategy that merely helps one sustain attention on a cue (Mace et al., 2017). In support of this notion, these processes appear to share two characteristics that set them apart from other retrieval processes: (1) They do not appear to involve additional cue elaboration, and (2) in both memories come to mind rather quickly, faster than the other strategies (Mace et al., 2017). We return to this point in the General discussion.

\section{The current study}

Our goal in the current study was to extend and gain further support for the multi-process retrieval theory. Here, we modify the multi-process retrieval theory to include three basic tenets, retrieval multiplicity, retrieval selectivity, and individual retrieval variation. The first idea (multiplicity) was discussed here and elsewhere (Mace et al., 2017), while the other two (retrieval selectivity and variation) we introduce here. As stated and reviewed, in retrieval multiplicity, many different types of retrieval processes are involved in the retrieval of autobiographical memories. Retrieval selectivity is the notion that one adjusts the retrieval processes to fit the retrieval circumstances such that different cues will evoke different types of retrieval processes, and, therefore, retrieval processes will vary in accord with the cueing environment (Barzykowski et al., 2019; Uzer, 2016; Uzer \& Brown, 2017). Retrieval variation is the notion that insight into the retrieval process will differ across individuals and across the lifespan. Thus, because of differing levels of metacognitive awareness, strategy use, in general, will vary across individuals and the lifespan (Barzykowski \& Staugaard, 2018; Barzykowski et al., 2019). Our focus in the present study was on retrieval multiplicity and retrieval selectivity.

Retrieval multiplicity is at the heart of the multi-process view as it asserts that autobiographical memory retrieval can occur in a variety of different ways (Mace et al., 2017; Uzer et al., 2012), a concept that, as discussed, can possibly be extended to all forms of declarative memory retrieval (e.g., semantic memory). Here, we were interested in showing that at least one additional retrieval strategy, visual imagery, is also at work in the recall of past experiences. In visual imagery (hereafter called visualization), individuals try to recall an event by attempting to visualize the scene or other visual 
aspects of the event. Such a strategy seems natural to autobiographical memory recall, given the visual foundation of such memories (a long-held and widespread position in autobiographical memory, e.g., Brewer, 1986; Rubin, 1998, 2006). Nevertheless, visualization strategies were not reported in Mace et al. (2017). However, we have observed visualization strategies in unpublished studies conducted in our lab. In addition to visualization, we have also observed other types of retrieval strategies in these studies that also weren't reported in Mace et al. (2017), though they occurred rather infrequently. Thus, in addition to visualization, we also believe that other retrieval strategies are potentially operative in the recall of autobiographical memories (discussed more fully in Experiments 1 and 2).

With respect to retrieval selectivity, as stated, we believe that retrieval strategies vary in accord with the retrieval requirements. For example, cues that do not contain lifetime period or other temporal information (e.g., going shopping) should be more likely to evoke hierarchical and temporal retrieval strategies than those that specify such information (e.g., your 16th birthday). Thus, retrieval selectivity should naturally interact with retrieval multiplicity, as the strategy that one uses will vary with the nature of the retrieval cues. While individuals may sense variation in the retrieval environment, and adjust their strategies accordingly, we also believe that retrieval selectivity is driven implicitly (i.e., outside of one's awareness). However, in this study we were focused solely on the base premise of retrieval selectivity, and to that end, we set out to demonstrate that both hierarchical and temporal retrieval would vary in accord with the presence and absence of lifetime and temporal information in retrieval cues.

We investigated retrieval selectivity and multiplicity across two experiments. In both experiments we used the retrievealoud procedure (Mace et al. 2017; Uzer et al., 2012). Experiment 1 investigated the possibility that retrieval strategies will vary with cue type, and that visualization, and possibly other strategies, may be used when one is trying to recall the past. Experiment 2 further investigated the retrieval multiplicity question, following up on findings reported in Experiment 1.

The general goal of this study was to further illuminate and document autobiographical memory retrieval strategies. By showing that there are multiple retrieval processes involved in the service of recalling past episodes, one is able to demonstrate that autobiographical memory retrieval is highly versatile and flexible. We hope that the data supporting such a proposition will spark a number of additional research agendas. For example, as noted above, it would be illuminating to learn if such retrieval flexibility is a function of declarative memory or is limited to autobiographical memory. Additionally, and perhaps ultimately, it is important to learn how, apart from their different mental content, each of the retrieval strategies may differ or function similarly to bring about memories. We return to these points in the General discussion.

\section{Experiment 1}

In Experiment 1, we combined a phrase-cue autobiographical memory task with the retrieve-aloud procedure (Mace et al. 2017; Uzer et al., 2012). As discussed previously, the retrievealoud procedure requires participants to verbalize everything that they are thinking while they are trying to recall specific experiences matching the retrieval cues. To test our retrievalselectivity hypothesis, we used two types of retrieval cues, lifetime period specific, and lifetime period nonspecific cues. Lifetime period specific cues refer to a specific lifetime period (e.g., remember your 16th birthday; high-school prom; first day of college), while lifetime period nonspecific cues (e.g., listening to music; going shopping) can refer to any lifetime period. In addition, these cues also vary along the general temporal dimension. For example, lifetime period specific cues might refer to the day, season, month, or other generic temporal markers (e.g., graduation was on a Saturday, in June, first day of college was in the fall or spring semester, etc.). Thus, given that temporal and lifetime period information is present in lifetime-period specific cues, the temporal and hierarchical retrieval strategies should occur less frequently with these cues, relative to lifetime-period nonspecific cues, as there would be little point in generating temporal or lifetimeperiod cues. Contrariwise, because such information is generally lacking with lifetime-period nonspecific cues, it would make sense to attempt to generate it in the hopes of retrieving a memory. In sum, then, we predicted that temporal and hierarchical retrieval strategies would occur more with lifetimeperiod nonspecific cues and less with lifetime-period specific cues. In the latter case, we predicted that with the decline of temporal and hierarchical retrieval strategies all other strategies would increase in frequency. The expectation that all other strategies would increase in this case was predicated on the base assumption of the retrieval-multiplicity hypothesis (i.e., that retrieval strategies are typically various).

Regarding retrieval multiplicity in general, based on the theoretical rationale and informal observations discussed in the Introduction, we expected that visualization would emerge as a retrieval strategy in the current experiment. Also based on informal observations, we also anticipated that other strategies might emerge. These strategies involved semantic associations (i.e., generating semantic information that might be related to an event), and using other known events to recall a target event. However, because these two strategies were observed to occur very infrequently and sporadically, and because of the possibility that other strategies might emerge, 
we classified all additional strategies as "other" in the current experiment.

\section{Method}

\section{Participants}

The participants were 36 undergraduate students from Eastern Illinois University, who participated in exchange for course credit. The age range of the participants was from 18 to 28 years $(M=19.6$ years), with 26 females and ten males.

\section{Materials and procedure}

The cue set for the autobiographical memory task consisted of 16 phrase cues, including two practice cues (see Appendix 1 for the complete set). Of the 14 target cues, seven were lifetime-period (LTP)-nonspecific cues, as they did not refer to a particular lifetime period (e.g., listening to music), while seven were LTP-specific cues, as they referred to specific lifetime periods (e.g., high-school prom).

We should note that the cues used in the LTP-specific condition can also be seen as representative of special events, transitions (Brown, et al., 2012), and first-time experiences (see Appendix 1). As special events, transitions, or first-time experiences, these cues contain known lifetime period information and temporal information (e.g., "your 16th birthday" was when you were a teenager, in high- school, lived in city X, the month of January, in winter, etc.). Thus, this type of information naturally co-varies with these cue types, and such cue categories should be among the cue types that are least likely to evoke the hierarchical and temporal retrieval strategies in everyday life.

Regarding the procedure, participants were told that they were to recall specific memories in response to phrase cues that were to be read aloud to them one at a time (the concept of specific autobiographical memories, episodic memories, was explained to them). They were further told that they were to perform the task out loud, verbalizing everything in their minds while they were trying to recall a memory, ultimately including the memory. Participants were then read the two practice cues one at a time and were instructed to retrieve memories according to the protocol. Once they demonstrated understanding of the procedure, the experiment commenced with the experimenter reading the phrase cues one at a time, with cue changes occurring only when participants responded with a memory or if they indicated that they had no memory for a cue. In cases where participants terminated a response with a general memory, they were asked to provide a specific memory. The cues would alternate between LTP-specific and LTP-nonspecific cues, with half the subjects receiving an LTP-specific cue on the first trial, half an LTP-nonspecific cue on the first trial. The experimenter took written and audio records of the verbalizations on a $\mathrm{PC}$, and all participants were tested individually (retrieval times were extracted from the audio recordings).

\section{Categorization method}

The retrieval interval was deemed as the time immediately following the reading of the cue and the utterance of a specific autobiographical memory. These intervals were categorized by two independent judges. The judges first placed intervals into two broad categories, "strategic" and "non-strategic." Intervals were deemed strategic if participates had verbalized any mental content, while they were deemed non-strategic (or direct retrieval) if the interval was marked by silence, following Mace et al. (2017) and Uzer et al. (2012). Strategic responses were further placed into six categories: hierarchical, repeating, temporal, visualization, mixed, and other. Verbal responses were deemed repeating if participants said the cue out loud one or more times without mentioning any other type of information (e.g., lifetime period information). Responses were deemed hierarchical if participants mentioned lifetime periods and/or general events and summary memories prior to retrieving a specific autobiographical memory. Utterances were deemed lifetime period or general/summary information only if the utterance had conformed to Conway's (2005) definitions of them. For example, for a lifetime period designation a participant had to mention the requisite abstract/personal, extended-event or other general-event information, such as when I was in the sixth grade, when we lived in Chicago, or on Michigan Avenue, etc. Both full hierarchical (i.e., lifetime period to general-event to specific-memory sequences) and truncated hierarchical (i.e., lifetime period to specific memory sequences or general event to specific memory sequences) verbalizations were marked as hierarchical retrieval. Verbalizations were deemed temporal if participants mentioned generic temporal markers in the interval, such as time of day, day of the week, month, year, seasons (e.g., summer), and other common/generic temporal markers (e.g., an academic semester). For example, they might involve I listen to music on weekends, or in the spring semester I go hiking. Often, temporal strategies manifest as searches through a mental calendar or diary (e.g., when did I do this last, last week, weekend, or month?). The temporal designation differed from the hierarchical designation in that it was assigned only if the utterance concerned generic temporal information, which was clearly not personal, such as a lifetime period or a summary event. Responses were marked visualization if participants verbalized their attempts to visualize the scene or other physical aspects of an event. For example, I see, or am imagining, the hall where the prom was, or I'm imaging the shopping mall where I shop a lot, etc. The mixed category was used in cases where participants verbalized two or more of the above strategies (e.g., a participant repeated the cue one or 
more times and subsequently mentioned lifetime period information). In these cases, participants had to start with one strategy and then switch to another, and perhaps another, and so forth, before they had reported a memory. The other category was used if participants used a strategy not specified above, and such a strategy was subsequently labeled according to its most salient features.

The two judges were fully trained in the coding process, which included familiarity with the literature on hierarchical retrieval and the hierarchical knowledge structures of the selfmemory system (e.g., Conway, 2005). All disagreements between them were settled through discussion. All attempts to retrieve memories, whether successful or not, were coded by the judges and included in the analyses below. The Kappa statistic indicated high agreement between the judges, $K=.83, S E=.03$.

\section{Results and discussion}

We first analyzed performance on the phrase-cue task in terms of memory production success, and general or summary memory production. This analysis showed that participants were able to generate autobiographical memories in response to the cues on $95 \%$ of all trials (the memory production rate was equal across cue type). On 5\% of these trials, participants terminated their retrievals with a general or summary autobiographical memory (73\% of general memory production was with LTP-nonspecific cues). When prompted to alternatively respond with a specific autobiographical memory, participants generated specific memories on $62 \%$ of these trials. An analysis of retrieval times for the two cue types showed that the average time for LTP-specific cues was $5.57 \mathrm{~s}(\mathrm{SD}=3.36)$, while it was and $5.47 \mathrm{~s}(\mathrm{SD}=4.03)$ for LTP-nonspecific cues $(t<1.0)$. We did not further analyze the retrieval-latency data, as the within-subject nature of the design produced very uneven samples (see Table 1; for retrieval-latency findings, see Barzykowski et al., 2019; Harris et al., 2015; Mace et al. 2017; Uzer et al., 2012). We also examined the data for possible order effects, and none were found. Overall, then, these analyses show that memory production, including specific memory production, was quite substantial, and retrieval time did not differ between the cue types.

Turning to the main questions of interest, Table 1 shows the results broken down by cue type. As can be seen in the table, the participants used all of the strategies enumerated above, including visualization, as well as strategies otherwise not previously identified (listed as "other," discussed below). The results were subjected to a two-way (retrieval strategy $x$ cue type) dependent-samples analysis of variance (ANOVA). The ANOVA indicated a significant main effect for retrieval strategy, $F(6,210)=39.44, M S E=5.93, p<001, \eta_{\mathrm{p}}{ }^{2}=.52$, a significant interaction between retrieval strategy and cue type, $F(6,210)=5.38, M S E=1.27, p<001, \eta_{\mathrm{p}}{ }^{2}=.13$, and no effect for cue type $(F<1.0)$. Follow-up on the interactive effect with Fisher's least significant difference (LSD) statistic revealed that hierarchical and temporal strategies were significantly higher with LTP-nonspecific cues relative to LTP-specific cues $(L S D=0.06$, see Table 1), consistent with our prediction on the retrieval selectively hypothesis. Other than hierarchical and temporal retrieval, no other strategies differed significantly across cue type, with all of them increasing in the LTPspecific cue condition, also consistent with our prediction (see Table 1).

An additional analysis of the hierarchical retrieval strategy across cue types showed that none of these retrievals in the LTP-specific cue condition contained the generation of lifetime period knowledge (i.e., all were general-to-specific memory sequences), while more than half of these same retrievals in the LTP-nonspecific cue condition contained lifetime period knowledge generation (i.e., $57 \%$ were either full hierarchical strategies or they were LTP-to-specific memory sequences). Truncated hierarchical retrieval strategies have been reported elsewhere (Mace et al., 2017), and the pattern of truncated hierarchical strategies observed across the cue conditions is consistent with our retrieval selectivity notion, as one would not predict that lifetime period knowledge would be generated in the LTP-specific cue condition.

We also further analyzed other and mixed retrieval strategies. The other category contained two distinct types of retrieval strategies, semantic memory generation (where

Table 1 Mean proportions of the retrieval strategies for lifetime-periodspecific and -nonspecific cues in Experiment 1 (standard deviations in parentheses)

Retrieval strategy $N$ Mean usage Mean retrieval time (in seconds)

Lifetime-period-specific cues

$\begin{array}{llll}\text { Direct } & 34 & .53(.31) & 3.58(1.23) \\ \text { Hierarchical } & 6 & .03(.08) & 9.53(3.36) \\ \text { Mixed strategies } & 9 & .06(.12) & 11.67(3.51) \\ \text { Repeating } & 26 & .25(.26) & 4.99(3.37) \\ \text { Temporal } & 11 & .05(.08) & 10.22(6.21) \\ \text { Other strategies } & 7 & .03(.04) & 6.55(3.01) \\ \text { Visualization } & 9 & .05(.11) & 9.87(2.10) \\ \text { Lifetime-period-nonspecific cues } & \\ \text { Direct } & 29 & .50(.31) & 3.81(1.19) \\ \text { Hierarchical } & 15 & .10(.14) & 9.60(3.89) \\ \text { Mixed strategies } & 3 & .02(.07) & 10.64(4.91) \\ \text { Repeating } & 20 & .20(.26) & 5.21(3.08) \\ \text { Temporal } & 22 & .16(.17) & 8.81(4.17) \\ \text { Other strategies } & 2 & .01(.04) & 6.33(2.88) \\ \text { Visualization } & 3 & .01(.03) & 8.67(7.02)\end{array}$

Note: Proportions were calculated out of the total memories retrieved, or attempted, for each participant 
participants generated related semantic information, such as songs or rock bands they had liked at the time, representing $61 \%$ of the total other category, slightly more than $1 \%$ of all retrievals), and other event generation (where participants generated other events to recall the target event, $39 \%$ of the total other category, under $1 \%$ of all retrievals). The semanticmemory-generation strategy tended to favor the LTP-specificcue condition (67\%, LTP-specific cues; 33\% LTP-nonspecific cues), while the other-event generation occurred equally across both conditions.

Concerning the mixed retrieval strategies, analyses of these retrievals showed that they all involved two-strategy combinations of four different types: repeating, semantic memory generation, temporal, and visualization. Combinations involving semantic memory generation appeared only in the LTPspecific-cue condition, while combinations involving the temporal strategy appeared only in the LTP-nonspecific-cue condition. Combinations involving visualization represented most $(90 \%)$ of the mixed retrieval strategies in the LTPspecific-cue condition, while all of the mixed retrieval strategies in the LTP-nonspecific-cue condition contained visualization.

Thus, the results of the strategy by cue-type interaction were consistent with our prediction concerning retrieval selectivity. Both the hierarchical and the temporal strategies were significantly increased in the LTP-nonspecific-cue condition relative to LTP-specific, where they occurred at a very low rate and were used by fewer individuals (see Table 1). The obvious explanation for this variation is lifetime period or temporal information could be generated with LTPnonspecific cues to retrieve a memory, but doing so with LTP-specific cues would be a pointless, unnecessary exercise. This point is sharpened by the complete lack of the lifetime period stage in hierarchical retrievals in the LTP-specific-cue condition. Overall, then, these results suggest that this type of variation exists in autobiographical memory retrieval in everyday life. The more lifetime period or temporal information a retrieval cue contains, the less likely it is that one will use a hierarchical or temporal strategy.

As mentioned earlier, the cues used in the LTP-specific-cue condition have a number of properties (e.g., first-time experiences, transitions). We believe that these are among the cue types in everyday life where one is less likely to use hierarchical or temporal strategies, as they inherently contain such information. While it is possible that these cues may have exerted other influences (e.g., produced more emotionally impactful memories), such influences independently did not appear to affect our data. If the cues in the LTP-specific-cue condition had exerted an effect on retrieval (e.g., in terms of greater cue specificity), then one might expect faster retrieval times and possibly a significant increase in direct retrieval, with significant decreases in all of the retrieval strategies.
None of these effects were found, as retrieval times did not vary across cue conditions, and all strategies, other than hierarchical and temporal, registered an increase, albeit non-significant, in the LTP-specific-cue condition (consistent with the concept of retrieval multiplicity).

Regarding additional retrieval strategies, as predicted, visualization did emerge as a strategy in the current experiment. As noted at the outset, we have observed visualization in unpublished observations in our lab. Also, as a strategy, it seems that visualization would occur commonly with autobiographical memories. However, the data obtained here do not seem to reflect this idea, as visualization occurred at a relatively low rate (.03 overall, while higher with LTP-specific cues, though the result across cue types was not significant, see Table 1). Perhaps the rate observed here does not accurately reflect the prevalence of the visualization strategy in everyday life, as visualization is probably difficult to verbalize, and therefore the retrieve-aloud task may have underestimated it (we address this possibility in the next experiment). Nevertheless, apart from these considerations, the appearance of this strategy in the results supports the retrieval-multiplicity hypothesis.

The retrieval-multiplicity hypothesis was also supported by the appearance of two other strategies in retrievals categorized as other. As mentioned earlier, we have noted both semanticmemory and other autobiographical-event generation in unpublished observations in our lab. How common such strategies might be in everyday life is a question open for future research. For the present purposes, we note their role in supporting retrieval multiplicity, as they potentially expand the number of different retrieval processes that can be used to recall autobiographical memories. We follow up on them, and visualization, in the next experiment.

\section{Experiment 2}

The goal of Experiment 2 was to see if visualization, as well as the other novel retrieval strategies reported in Experiment 1, would be observed again with the retrieve-aloud procedure, thereby garnering additional support for the retrieval multiplicity theory. We employed the retrieve-aloud procedure again, but with slightly modified instructions and a different cue set. We added instructions to enhance verbal reporting, as we believe that the visualization strategy may have been under-reported in Experiment 1, given the potential difficulties in reporting a non-verbal mental process like visualization. The cue set was comprised of LTP-nonspecific cues, which as in Experiment 1 referenced potentially mundane experiences (e.g., listening to music; going shopping). We made this change because we were not interested in manipulating the cue-type variable again, and we were particularly interested in seeing if the novel strategies reported in 
Experiment 1 would be obtained with this cue set (i.e., solely LTP-nonspecific cues).

\section{Method}

\section{Participants}

The participants were 21 undergraduate students from Eastern Illinois University, who participated in exchange for course credit. The age range was from 18 to 21 years $(M=18.85$ years), with 16 females and five males.

\section{Materials and procedure}

The materials for the autobiographical memory task consisted of 12 general phrase cues, which mostly referred to mundane experiences (e.g., listening to music) without reference to specific lifetime periods (see Appendix 2).

Regarding the procedure, it was the same as in Experiment 1 , except that we added one additional instruction that was designed to enhance reporting, particularly when strategies might involve visualization. The instruction was inserted after the general instructions about the retrieve-aloud task were delivered. It was as follows: "While you are trying to recall a memory, it is important to say everything that is going through your mind. Even if all you have is pictures (or images) in your mind, tell us about them." As in Experiment 1, once the task instructions were delivered, practice trials commenced. After the practice trials were completed, the new instruction was repeated.

\section{Categorization method}

The categorization method was the same as in Experiment 1, with the exception that the strategies appearing in the other category designation in Experiment 1 were added to the list of retrieval categories for Experiment 2. They were semanticmemory generation, defined as generating semantic information to assist in event retrieval, and other-event generation, defined as retrieving another event memory in an attempt to retrieve the target event. For the strategy to be classified as semantic-memory generation, participants had to use generic information as apparent cues to retrieval (such as naming rock bands in order to remember a concert or music memory), while in other-event generation, participants had to retrieve another episodic memory in order to retrieve the target episodic memory. In the last case, the other event was a memory for an episode that did not match the cue, but apparently had some relationship to it according to the participant. As in Experiment 1, all attempts to retrieve memories, whether successful or not, were coded by the judges and included in the analyses below. Agreement between the judges was excellent, $K=.86, S E \mathrm{~s}=.02$.

\section{Results and discussion}

An analysis of overall performance on the phrase-cue task showed that participants were able to generate autobiographical memories in response to the cues on $93 \%$ of all trials. On $3 \%$ of these trials, participants terminated their retrievals with a general or summary autobiographical memory. When prompted to alternatively respond with a specific autobiographical memory, participants generated specific memories on $37 \%$ of these trials. For the same reason cited in Experiment 1, we did not analyze the retrieval-latency data.

The results of the retrieve-aloud task are presented in Table 2. As can be seen in the table, all of the strategy categories observed in Experiment 1 were observed in the current study, including visualization, semantic-memory generation, and other-event generation. No novel strategies were found (i.e., those that would be classified as "other"). We submitted the results in Table 2 to a dependent-samples ANOVA. The ANOVA indicated a significant difference among the strategies, $F(6,140)=22.71, M S E=0.02, p<001, \eta_{\mathrm{p}}{ }^{2}=.53$. Follow-up with the LSD statistic $(\mathrm{LSD}=.09)$ showed that direct retrieval was significantly more frequent than all other retrieval types; hierarchal, temporal, repeating, and visual did not differ; visual, mixed, semantic-memory generation, and other-event generation did not differ; while hierarchal, temporal, and repeating were significantly more frequent than mixed, semantic-memory, and other-event generation.

In summary, Experiment 2 showed that participants used hierarchal, temporal, and repeating strategies as observed, generally, in Experiment 1 and in Mace et al. (2017). As observed in Experiment 1, participants also used the visualization, semantic-memory generation, and other-event generation strategies. Visualization did occur at a higher frequency relative to Experiment 1 (i.e., .08 vs. .03), and perhaps the added instruction enhanced reporting with this strategy. Still, it is possible that the observed frequency is an underestimate of what may occur in everyday life, as the instructions probably did not entirely eliminate the difficulty of reporting such a process. We discuss this and other possibilities in the General discussion.

Overall, then, the results appear to confirm that visualization, semantic-memory generation, and other-event generation strategies are retrieval strategies that individuals use to recall the past, perhaps somewhat more commonly in the case of visualization. The result, therefore, bolsters and extends the basic notion that retrieval in autobiographical memory is multiplicitous, as it confirms a number of additional retrieval strategies in the autobiographical memory-retrieval repertoire.

\section{General discussion}

In this study, we tested the multi-process retrieval account of autobiographical memory. We set out to test two tenets of this 
Table 2 Mean proportions for the retrieval strategies on lifetime-period-nonspecific cues in Experiment 2 (standard deviations in parentheses)

\begin{tabular}{llll}
\hline Retrieval strategy & $N$ & Mean usage & Mean retrieval time (in seconds) \\
\hline Direct & 21 & $.46(.21)$ & $3.73(1.17)$ \\
Hierarchical & 16 & $.16(.12)$ & $8.61(2.49)$ \\
Mixed strategies & 8 & $.01(.03)$ & $7.49(4.13)$ \\
Repeating & 12 & $.14(.20)$ & $5.03(1.83)$ \\
Temporal & 13 & $.12(.15)$ & $8.29(1.78)$ \\
Other event generation & 5 & $.02(.04)$ & $4.56(2.85)$ \\
Semantic memory generation & 2 & $.01(.03)$ & $5.60(2.07)$ \\
Visualization & 7 & $.08(.13)$ & $7.98(5.43)$ \\
\hline
\end{tabular}

Note: Proportions were calculated out of the total memories retrieved, or attempted, for each participant

account - retrieval multiplicity and retrieval selectivity. In Experiments 1 and 2 we observed participants using numerous types of retrieval strategies to recall past experiences. Some of these strategies (e.g., repeating) were reported elsewhere (Mace et al. 2017), and some (e.g., visualization) were newly observed in this study. Taken as a whole, these observations both reinforce and extend the notion of retrieval multiplicity. In Experiment 1 we also found that retrieval strategies varied across cue types, such that hierarchical and temporal retrieval strategies were significantly more common with LTP-nonspecific cues relative to LTP-specific cues. This observation supports the notion of retrieval selectivity, as the variation of these two strategies was perfectly consistent with the information contained in the two different cue types. We begin our discussion of these findings with the retrieval multiplicity.

Retrieval multiplicity is the fundamental premise of the multi-process retrieval account, as this premise states that retrieval from autobiographical memory involves a diverse set of processes. Looking across the two experiments reported here, we now document that eight different retrieval processes can be used to construct autobiographical memories. The processes ranged from the seemingly automatic (direct retrieval) to the less (e.g., repeating) or more (e.g., hierarchical) sophisticated retrieval strategies. Each of the retrieval processes appears to be distinctly different, and, therefore, each appears to represent a different way that autobiographical memories can be retrieved. For example, one might merely repeat the cue until a memory comes to mind, visually imagine the scene, or recall other events. With the exception of the repeating strategy, which may be an extension of direct retrieval (see Mace et al., 2017, and below), each of the strategic processes can be seen as an indirect retrieval process, where one generates different forms of related knowledge (or cues) in order to retrieve the target episodic memory (cf. Conway, 2005; Uzer et al., 2012). Thus, one may generate general temporal cues (e.g., the days of the week), lifetime period or general event knowledge (Haque \& Conway, 2001), related events or general knowledge, or the likely visual scene of the event. Once generated, each of these knowledge forms is thus able to map directly onto the target autobiographical memory. Two of the strategies (temporal and visualization), in addition to hierarchical retrieval, appear to rely on access to knowledge at the general-event layer of autobiographical memory (Conway, 2005). That is, in visualization the generated physical elements of an event (e.g., a scene or an individual) represent knowledge held at the general-event level of autobiographical memory, according to Conway's theory. In the temporal strategy, generated temporal markers may at times be associated with summary memory structures (i.e., repeated-event knowledge), knowledge that also represents general-event-level knowledge. Thus, one way to view the temporal and visualization strategies is to see them as variants of hierarchical retrieval, or at least strategies that make use of general autobiographical knowledge (i.e., some element of the autobiographical memory hierarchy). The same could be said for semanticmemory generation, as here individuals appear to be generating semantic memories that have personal meaning, or even other-event generation as this appears to rely on memories held at the episodic layer.

As mentioned, we believe that visualization strategies might occur more in everyday life, but that was not evident here, owing to the potential difficulty in verbalizing nonverbal processes. We should also note that there may have been task factors that may have directly suppressed the selection of a visualization strategy. For example, perhaps visualization is more likely to occur when retrieval is somewhat demanding. The cues used in Experiments 1 and 2 were relatively specific phrase cues, which appeared to induce retrieval fairly quickly (e.g., typically under $10 \mathrm{~s}$; see Tables 1 and 2). If visualization is more likely under demanding retrieval circumstances, then our cues may have reduced its likelihood as retrieval with them may not have been sufficiently demanding. In some cases, scene construction may have occurred so quickly that participants did not experience the process as involving a strategy.

Before turning to the retrieval-selectivity results, we should note an argument about the repeating strategy put forward in 
Mace et al. (2017). There, it was argued that repeating might be an extension of direct retrieval, as the goal of this strategy is to hold the cue in mind by repeating it until a memory surfaces. Thus, repeating is like direct retrieval because one does not generate additional retrieval cues or autobiographical memory content, but instead focuses on the retrieval cue with the knowledge that this will result in direct retrieval. Unlike direct retrieval, it was argued that individuals select this process as a strategy because they have some knowledge of direct retrieval (i.e., that mere thought can produce memories) and that sustained thought on a retrieval cue will likely be successful. Support for this view was found in the second experiment of Mace et al. (2017), where a group of participants was instructed to exclusively use the repeating strategy, and their results showed shortest retrieval latencies relative to hierarchical and temporal strategy groups (see also Tables 1 and 2, this study).

The results of this study also found support for the notion of retrieval selectivity. Consistent with this idea, we observed a significant increase in the use of hierarchical and temporal strategies for LTP-nonspecific cues relative to LTP-specific cues in Experiment 1. This result suggests that the retrieval process was adjusted to include or exclude these strategies, given that temporal knowledge was present in one set of cues while absent in the other. We believe that this is good evidence in support of the retrieval selectivity, as the data showed that strategies adjusted to the cuing environment in sensible ways. We also believe, as discussed, that the pattern observed in Experiment 1 is likely to occur in everyday life. When cues contain lifetime period or temporal information, the hierarchical and temporal strategies will have a very low probability of occurrence. When, on the other hand, they lack such information, these strategies may be as probable as most other retrieval strategies. Additionally, certain circumstances may make these strategies more likely than others. For example, when trying to remember a rare event, such as in the case of the cue "have you ever been to a Malaysian restaurant," one might use a hierarchical strategy (e.g., perhaps I was when I lived in New York City, use to visit Europe, etc.), as this might be a circumstance when generating lifetime period cues are imperative to retrieval. While the data from Experiment 1 suggest that such variations are likely with these two strategies, we should note that retrieval selectivity should apply to all autobiographical memory-retrieval processes. Evidence that other retrieval processes are subject to variation can be found in Uzer (2016) and Uzer and Brown (2017), where direct retrieval was shown to vary as a function of cue type.

Thus, the evidence for retrieval multiplicity and selectivity obtained here demonstrates that autobiographical memory retrieval is enormously flexible. This property of autobiographical memory retrieval may be because of the richness and complexity of autobiographical memories. Rubin's account of autobiographical memory may offer some additional insights here (e.g., Rubin, 2012). In his basic systems account, he argues that numerous systems are involved in the construction of autobiographical memories (e.g., vision, audition, spatial imagery, language, emotion, narrative, and many others; see Rubin, 2012, 2019). Given the complexity and variety of the many systems involved, it is reasonable to imagine that a concomitant set of different processes could be involved in the construction of autobiographical memories. Thus, because each system is a different knowledge base/ domain and each contributes to autobiographical knowledge, each may allow for access to autobiographical memories in one (or more) ways.

As noted earlier, the multi-process retrieval account might not be limited to autobiographical memory, as at least one study has shown that different strategies are used to recall semantic memories (Minakova \& Falikman, 2011). For example, Minakova and Falikman found that participants used either a semantic- (i.e., using general knowledge cues) or an episodic- (i.e., using episodic cues) retrieval strategy when trying to recall general semantic knowledge. Although that study only documented that two types of retrieval processes were used to recall semantic memories, given the complexity and variety of semantic knowledge, it is reasonable to imagine that more retrieval processes might be used in the recall of semantic memories. Perhaps, as in the case of autobiographical memory, retrieval-process diversity in semantic memory overlaps with the many different types of systems and knowledge domains that exist in semantic memory. For example, different strategies might be used in the recall of linguistic information, general knowledge, common names or faces, and so forth. If this account of retrieval in semantic memory is true, then retrieval multiplicity may be a general property of declarative memory, rather than limited to autobiographical memory.

At another level, as noted earlier, the multi-process retrieval account is neutral with respect to the constructed (i.e., memory reconstruction) versus retrieved (i.e., retrieval of stable event units) debate in autobiographical memory (e.g., Conway, 2005; Haque \& Conway, 2001; Uzer et al., 2012). We believe that our data can fit either a construction (Conway, 2005) or a retrieval (Uzer et al., 2012) account of autobiographical memory production (see also Harris et al., 2015). And, as noted throughout, while the multi-process view takes the position that each strategy (or retrieval process) represents a different underlying way in which autobiographical memories produced, we believe that one (or two) base processes (e.g., spreading activation) may underlie all memory production. For example, whether one is modeling hierarchical retrieval or a visual imagery strategy, each could be explained with spreading activation (e.g., lifetime period memories spread to general-event memories, or generic images of scenes spread to specific autobiographical memories involving such scenes). Nevertheless, the multi-process retrieval view is 
neutral on the different possibilities, as the account is unable to distinguish between different underlying retrieval mechanisms (e.g., spreading activation vs. as more active processes, such as in MINERVA 2, Hintzman, 1986).

This study is not without its limitations. For instance, while we believe that the presence or absence of lifetime period and temporal information in the cues used in Experiment 1 drove the variability of the hierarchical- and temporal-retrieval strategies, it is possible that other factors were also at work. As mentioned, the specificity of LTP-specific cues may have helped to narrow the selection of retrieval strategies. In addition, the fact that some cues were first-time, unique, or transitional experiences may have also helped narrow the focus on strategies. Notwithstanding our argument concerning the lack retrieval-latency differences between LTP-specific and non-specific cues, we cannot rule out the possibility that other cue properties contributed to the results of Experiment 1. However, despite this possibility, we think it is important to note that some property, or set of properties, drove retrieval-strategy selection, and this is consistent with and evidence of general retrieval-strategy selectivity.

In addition, the retrieve-aloud methodology has some limitations. As noted, temporal strategies may at times overlap with hierarchical retrieval. In such cases, it would be difficult for the methodology to distinguish between a pure temporal strategy and one that may verge on or in fact be a variant of hierarchical retrieval. Thus, one cannot see the methodology as providing the most precise and accurate measure of the prevalence of such strategies. Nevertheless, with respect to hierarchical and temporal retrieval, at the very least the approach has been able to distinguish between pure hierarchical strategies (i.e., following the lifetime period, general event, and episodic memory pathway), and strategies that produce episodic memories by accessing or generating cues from the hierarchy. In addition to this possibility, the methodology may not detect unique forms of hybrid strategies. That is, the visualization strategy may at times accompany and contribute to other strategies, such as direct retrieval or the repeating strategy. In such a notion, two strategies are combining during retrieval to produce memories, a process different from mixed strategies, as the latter form is simply strategy switching. We think that the possibility of hybrid strategies is intriguing as they add another dimension to the retrieval problem, and future studies may want to direct their efforts at uncovering such possibilities. The idea of hybrid strategies also highlights the possibility that covert strategies may at times underlie direct or repeating retrieval. Future work into hybrid strategies may uncover such possibilities. Finally, it is possible that visualization might sometimes be a consequence of retrieval (e.g., direct retrieval), but it is articulated in the retrieve-aloud approach in such a way as to confuse it with a strategy. If true, the question in these cases is does the generated image then play a role in the final retrieval of a specific memory.

In conclusion, this study extended and found further support for the multi-process view. As this approach is a relatively novel, there remain many open questions. For example, future work should be directed towards understanding how each of the retrieval processes observed here is able to produce autobiographical memories. As noted above, future research should also pursue the extension of the multi-process view to other forms of declarative memory, namely semantic memory. With respect to the retrieval selectivity, future work should focus attention on how other strategies might vary, as well as the role of explicit and implicit processes in such variation. As noted in the Introduction, internal factors are likely to play a role in retrieval selectivity. Given the vast literature on metacognition in memory and implicit processes in memory and cognition, it would be surprising if explicit and implicit processes were not factors (see reviews in Kihlstrom, 1987; Koriat \& Helstrup, 2007; Reber; 1993). Relatedly, with respect to the idea that retrieval might vary across individuals, there is much work to be done. While it is reasonable to imagine that retrieval strategies will vary across individuals (e.g., due to varying levels of meta-awareness) and the lifespan (e.g., due to increasing levels of meta-awareness with age), there currently is no direct evidence to support this basic proposition (but see other work on meta-awareness in autobiographical memory, e.gBarzykowski and Staugaard, 2018, Barzykowski, et al., 2019). Thus, the obvious first step for future work here is to obtain evidence that establishes this basic tenet.

\section{Appendix 1}

Phrase cues used in Experiment 1

Going to a Concert*

Going to a sporting event*

Doing yard work**

Your 16th birthday***

Listening to music**

First day of college***

Riding on a bus or train**

First day of High school***

Being at a wedding**

First day of Middle school***

Taking a hike or a walk**

High school prom***

Being at a barbeque**

Your 18th birthday***

Going shopping**

High school graduation ceremony***

Notes: * Indicates practice cue. ${ }^{* *}$ Indicates lifetime period nonspecific cue. $* * *$ Indicates lifetime period specific cue. 


\section{Appendix 2}

Phrase cues used in Experiment 2

Going to a Concert*

Going to a sporting event*

Being at a friend's house

Doing yard work

Taking a hike or a walk

Listening to music

Riding on a bus or train

Taking a car trip

Riding a bicycle

Taking a walk in the woods

Playing a game

Visiting a family member

Being at a party

Going shopping

Note: * Indicates practice cue

\section{References}

Ball, C. T., \& Little, J. C. (2006). A comparison of involuntary autobiographical memory retrievals. Applied Cognitive Psychology, 20, 1167-1179.

Barzykowski, K.J., Niedzwienska, A., Mazzoni, G. (2019). How intention to retrieve a memory and expectation that a memory will come to mind influence the retrieval of autobiographical memories. Consciousness and Cognition, 72, 31-48.

Barzykowski, K.J., \& Staugaard, S.R., (2016). Does retrieval intentionality really matter? Similarities and differences between involuntary memories and directly and generatively retrieved voluntary memories. British Journal Psychology, 107, 519-536.

Barzykowski, K.J., \& Staugaard, S.R., (2018). How intention and monitoring your thoughts influence characteristics of autobiographical memories. British Journal Psychology, 109, 321-340.

Berntsen, D. (1996). Involuntary autobiographical memory. Applied Cognitive Psychology, 10, 435-454.

Berntsen, D. (1998). Voluntary and involuntary access to autobiographical memory. Memory, 6, 113-141.

Brewer, W. F. (1986). What is autobiographical memory? In D. C. Rubin (Ed.), Autobiographical memory (pp. 25-49). Cambridge: Cambridge University Press.

Brown, N.R., Hansen, T.G.B., Lee, P.J., Vanderveen, S.A., \& Conrad, F.G. (2012). Historically defined autobiographical periods: Their origins and implications. In D. Berntsen, \& D.C. Rubin (Eds.), Understanding autobiographical memory (pp. 160-180). New York: Cambridge University Press.

Burt, C.D.B., Kemp, S., \& Conway, M. (2001). What happens if you retest autobiographical memory 10 years on? Memory \& Cognition, $29,127-136$.

Conway, M. A. (2005). Memory and the self. Journal of Memory and Language, 53, 594-628.

Conway, M.A., \& Pleydell-Pearce, C.W. (2000). The construction of autobiographical memories in the self-memory system. Psychological Review, 107, 261-288.

Haque, S., \& Conway, M.A. (2001). Sampling the process of autobiographical memory construction. European Journal of Cognitive Psychology, 13, 529-547.
Haque, S., Eka, J., Khan, R., \& Hasking, P. (2014). Autobiographical memory and hierarchical search strategies in depressed and nondepressed participants. BMC Psychiatry, 14, 310.

Harris, C.B., \& Berntsen, D. (2019). Direct and generative autobiographical memory retrieval: How different are they? Consciousness and Cognition, 74, 1-10.

Harris, C.B., O'Connor, A.R., \& Sutton, J. (2015). Cue generation and memory construction in direct and generative autobiographical memory retrieval. Consciousness and Cognition, 33, 204-216.

Hintzman, D.L. (1986). "schema abstraction" in a multiple trace memory model. Psychological Review, 93, 411-428.

Kihlstrom, J.F. (1987). The cognitive unconscious. Science, 237, 14451452.

Koriat A., \& Helstrup, T. (2007). Metacognitive aspects of memory. In S. Magnussen, \& T. Helstrup (Eds.), Everyday memory (pp. 251-274). New York: Psychology Press.

Mace, J.H. (2003). Study-test awareness can enhance priming on an implicit memory task: Evidence from a word completion task. American Journal of Psychology, 116, 257-279.

Mace, J.H. (2005). Priming involuntary autobiographical memories. Memory, 13, 874-884.

Mace, J.H., Clevinger, A.M., Delaney, D.M., Mendez, A.S., \& Simpson, S.H. (2017). Voluntary remembering: Elucidating the mental strategies used to recall the past. Applied Cognitive Psychology, 31, 156163.

Minakova, M. A. \& Falikman, M. V. (2011). Knowledge retrieval strategies: The case of an artificial domain. Journal of Russian and East European Psychology, 49, 55-67.

Norman, D.A., \& Bobrow, D.G. (1979). Descriptions: An intermediate stage in memory retrieval. Cognitive Psychology, 11, 107-123.

Reber, A. S. (1993). Implicit knowledge and tacit knowledge: An essay on the cognitive unconscious. New York: Oxford University Press.

Richardson-Klavehn, A., Gardiner, J. M., \& Java, R. I. (1994). Involuntary conscious memory and the method of opposition. Memory, 2, 1-29.

Roediger, H.L., III, \& McDermott, K. (1993). Implicit memory in normal human participants. In F. Boller \& J. Grafman (Eds.), Handbook of neuropsychology (Vol. 8, pp. 63-131). New York: Elsevier Science.

Rubin, D. C. (1998). Beginnings of a theory of autobiographical remembering. In C. P. Thompson, D. J. Herrmann, D. Bruce, J. D. Reed, D. G. Payne, and M. P. Toglia (Eds.), Autobiographical memory: Theoretical and applied perspectives (pp. 47-67). Mahwah, NJ: Erlbaum.

Rubin, D. C. (2006). The basic-systems model of episodic memory. Perspectives on Psychological Science, 1, 277-311.

Rubin, D. C. (2012). The basic systems model of autobiographical memory. In D. Berntsen, \& D.C. Rubin (Eds.), Understanding autobiographical memory (pp. 11-32). New York: Cambridge University Press.

Rubin, D. C. (2019). Placing autobiographical memory in a general memory organization. In J.H. Mace (Ed.), The organization and structure of autobiographical memory (pp. 6-27). New York: Oxford University Press.

Schacter, D.L. (1992). Priming and multiple memory systems: Perceptual mechanisms of implicit memory. Journal of Cognitive Neuroscience, 4, 244-256.

Schlagman, S., \& Kvavilashvili, L. (2008). Involuntary autobiographical memories in and outside the laboratory: How different are they from voluntary autobiographical memories? Memory \& Cognition, 36, 920-932.

Thompson, C.P., Skowronski, J.J., \& Betz, A.L. (1993). The use of partial temporal information dating personal events. Memory \& Cognition, 21, 352-360.

Tulving, E. (1983). Elements of Episodic Memory. Oxford: Clarendon. 
Uzer, T. (2016). Retrieving autobiographical memories: How different retrieval strategies associated with different cues explain reaction time differences. Acta Psychologica, 164, 144-150.

Uzer, T., \& Brown, N.R. (2017). The effect of cue content on retrieval from autobiographical memory. Acta Psychologica, 172, 84-91.

Uzer, T., Lee, P.J., \& Brown, N.R. (2012). On the prevalence of directly retrieved autobiographical memories. Journal of Experimental Psychology: Learning, Memory and Cognition, 38, 1296-1308.
Williams D.M., \& Hollan J.D. (1981). The process of retrieval from very long-term memory. Cognitive Science, 5, 87-119.

Williams, J.M.G., Chan, S., Crane, C. \& Barnhofer, T. (2006). Retrieval of autobiographical memories: The mechanisms and consequences of truncated search. Cognition and Emotion, 20, 351-382.

Publisher's note Springer Nature remains neutral with regard to jurisdictional claims in published maps and institutional affiliations. 